\title{
émulations
}

\section{Nicolas Charles - Enseignement supérieur et justice sociale. Sociologie des expériences étudiantes en Europe}

\author{
Magali Ballatore
}

Émulations - Revue de sciences sociales

2016, « Comptes rendus critiques, En ligne»

Article disponible à l'adresse suivante

https://ojs.uclouvain.be/index.php/emulations/article/view/7213

Pour citer cet article

Magali Ballatore, « Nicolas Charles — Enseignement supérieur et justice sociale. Sociologie des expériences étudiantes en Europe », Émulations, en ligne. Mise en ligne le 29 août 2016.

DOI : 10.14428/emulations.cr.023

Distribution électronique : Université catholique de Louvain (Belgique) : ojs.uclouvain.be

(c) Cet article est mis à disposition selon les termes de la Licence Creative Commons Attribution, Pas d'Utilisation Commerciale 4.0 International. http://creativecommons.org/licenses/by-nc/4.0/

Éditeur : Émulations - Revue de sciences sociales / Presses universitaires de Louvain https://ojs.uclouvain.be/index.php/emulations

ISSN électronique : $1784-5734$

UCL PRESSES

UNIVERSITAIRES

DE LOUVAIN 


\title{
Nicolas Charles - Enseignement supé- rieur et justice sociale. Sociologie des ex- périences étudiantes en Europe
}

\begin{abstract}
Magali Ballatore $^{1}$
Recensé : Nicolas Charles (2015), Enseignement supérieur et justice sociale. Sociologie des expériences étudiantes en Europe, Paris, La Documentation française (« Études \& recherche »).
\end{abstract}

Cet ouvrage se propose d'étudier les principes de justice dans l'enseignement supérieur de trois pays européens. Il s'inscrit très clairement dans la sociologie de l'expérience étudiante de François Dubet (2009), avec ses principes de justice (l'égalité, le mérite et l'autonomie), tout en s'inspirant également fortement de la typologie d'EspingAndersen (1999) pour comparer la France « corporatiste », la Suède « sociale-démocratique » et l'Angleterre « libérale ». Nicolas Charles part du constat que les comparaisons qualitatives de systèmes d'enseignement supérieur sont rares, sans pour autant citer les analyses comparées existantes et les études internationales relatives notamment aux politiques éducatives, aux stratégies des familles, aux curricula ou à la mobilité en Europe (entre autres Ballatore, 2002 ; 2010 ; Ballarino et al., 2009 ; 2010 ; Felouzis, Maroy, van Zanten, 2013 ; Garneau, 2008 ; Turner, 1960 ; van Zanten, Kosunen, 2013 ; Vitale, 1999 ; 2001). L’hétérogénéité des systèmes est approchée par une étude de cas sur des filières de divers segments des systèmes de formation supérieure dans des universités des capitales de ces pays. Deux filières sont choisies : l'une en économie et mangement que N. Charles nomme «formation prestigieuse », et l'autre en histoire, dite « de démocratisation ». Cette comparaison permet de saisir une certaine variance interne à chaque système.

Les modèles contrastés de financements des études selon les pays sont bien décrits, avec l'importance prise, notamment en Angleterre, par les prêts à remboursement contingent au revenu et par les bourses généreuses en Suède, qui peuvent éventuellement être complétées par des prêts, alors qu'en France seul $1 \%$ des étudiants financent leurs études grâce à un système de prêt. Cependant, le regard sur les statistiques provenant d'études comparatives, telles qu'Eurostudent, est assez peu critique. C'est peut-être ce qui amène l'auteur à dire que les étudiants les plus modestes semblent moins soutenus

${ }^{1}$ Etudiant en master 2 au Département de Sciences sociales de l'École normale supérieure, agrégé de sciences économiques et sociales. 
en France qu'en Angleterre et en Suède. En outre, on peut s'interroger sur le fait de classer les prêts parmi les « aides » reçues. En effet, en Angleterre, les étudiants à bas revenus sont surreprésentés parmi ceux qui manifestent la plus forte aversion pour l'emprunt et qui préfèrent avoir une activité rémunérée, alors qu'on interdit à leurs confrères d'Oxford de travailler au cours de l'année universitaire. De manière générale, cet ouvrage tend ainsi à se concentrer sur l'hétérogénéité des expériences étudiantes en France, tout en mettant en avant une certaine uniformité des expériences en Angleterre ou en Suède. L'aide des parents obtenue par les étudiants de l'école de commerce étudiée est, par exemple, considérée comme spécifique et liée au contexte de la capitale suédoise.

Dans le chapitre suivant, $\mathrm{N}$. Charles se penche sur la fonction sélective de l'enseignement supérieur et sur la méritocratie scolaire, qui jouit d'une légitimité très forte dans les sociétés occidentales. L’auteur rappelle que le mérite est principalement une affaire d'environnement social (Duru-Bellat, Meuret, 2009). Ainsi parler de « compétition équitable » est déjà un oxymore en soi. Les procédures d'évaluation du mérite sont présentées de manière successive comme des cas nationaux. $\mathrm{N}$. Charles voit en Angleterre une volonté latente d'évaluer davantage le potentiel des individus que leurs performances passées. Pourtant, comme en France, ce sont d'abord les notes du secondaire qui vont permettre aux étudiants de pouvoir demander l'inscription dans telle ou telle filière, tels ou tels établissements, qui sont explicitement classés des plus prestigieux ou moins exigeants. Par conséquent, ce que l'auteur appelle « l'individualisme du système » n'estil pas plutôt une plus grande autonomie laissée aux universités dans le choix de leurs candidats ? La spécificité, qui consiste à demander aux candidats d'exposer leurs motivations et leurs expériences personnelles, est une pratique que l'on retrouve également aujourd'hui, par exemple, dans les classes préparatoires spécifiques destinées aux enfants des zones d'éducation prioritaire en France (Pasquali, 2014). Finalement, on observe beaucoup de similitudes entre les discours et les pratiques des étudiants de filières sélectives en Angleterre et en France, en particulier le réflexe corporatiste qui les pousse à légitimer le processus de sélection (impartial à leurs yeux). Les grandes écoles sont ainsi, comme les vieilles universités anglaises, des institutions englobantes.

La Suède, quant à elle, considérerait plutôt le principe méritocratique comme un mal nécessaire, lui préférant l'idéal de la seconde chance, dans un contexte de marchandisation récente de l'école secondaire, qui crée de nouvelles inégalités (Palme, Hultqvist, 2009). Peut-on considérer l'introduction des «points de mérite » comme un tournant vers la valorisation des performances scolaires en Suède, étant donné qu'il existait déjà des mentions auparavant ? Une série de réformes, que l'auteur évoque justement, contribue en tout cas à transformer l'école secondaire supérieure en un espace de formation de plus en plus segmenté. Le modèle suédois est basé, d'une part, sur des programmes prédéfinis et, d'autre part, sur des "cursus libres », qui laissent un grand choix dans la construction des parcours. Ces évolutions et ces programmes ne sont pas sans rappeler les distinctions à l'intérieur des codes-séries de la typologie de Basile 
Bernstein (1975 : 191-222) et les deux voies qu'il distingue, l'une «pure », l'autre «impure » (disciplines provenant de domaines différents du savoir). Pendant longtemps, la première avait un statut supérieur en Angleterre, mais l'accroissement du nombre de programmes impurs (la seconde) permet de plus en plus d'absorber l'augmentation du flux d'étudiants, tout en maintenant le mythe de l'égale valeur de toutes les formations. C'est aussi dans un contexte où les ressources financières déclinent rapidement que les dirigeants des institutions ont commencé à embaucher cette idée de l'utilisation de la demande du consommateur (l'étudiant) comme une arme pour réclamer des fonds supplémentaires. Peut-on dire, dans le cas de la Suède, que les motifs du décloisonnement, du développement de voies moins pures et moins spécialisées, sont davantage pragmatiques qu'égalitaires ? L'auteur ne répond pas à cette question, qui aurait demandé une incursion dans la sociologie bernsteinienne. Pourtant, les évolutions qu'il décrit semblent mettre en exergue la croyance erronée que chaque choix par les étudiants peut permettre une variation seulement du sujet et non pas du niveau, car il existe des différences patentes entre les niveaux attendus dans les diverses options. C'est, par conséquent, une part de l'exercice des étudiants que de contrôler le curriculum caché, pour découvrir quelles options sont plus hautement considérées académiquement parlant, ou lesquelles requièrent le moins d'effort au regard de leurs ambitions et besoins.

L'Angleterre se distingue, en fait, de la Suède et surtout de la France, par l'essor des études à temps partiel, par le fait que les étudiants poursuivent très peu leurs études jusqu'au master et s'insèrent fréquemment dans des secteurs d'emploi qui ne sont pas directement en lien avec leurs domaines d'étude. Ce qui caractérise la France est, effectivement, comme le souligne $\mathrm{N}$. Charles, l'accès très limité des adultes aux formations ainsi que le poids des études initiales dans les parcours diplômants et professionnels des jeunes. On pourrait par exemple aussi ajouter la place qui y est accordée à l'écrit par rapport à l'oral. L'auteur se penche aussi dans cet ouvrage sur les modèles de décohabitation et les raisons de la «marche progressive vers l'indépendance » différenciée en Europe, qui sont à rechercher dans des modèles de mises en couple, de solidarité familiale et d'insertion professionnelle bien différents. En Italie, par exemple, les étudiants restent en moyenne plus longtemps inscrits à l'université, décohabitent tard et s'insèrent professionnellement beaucoup moins aisément sur le marché du travail que les Français ; ces écarts sont encore plus importants si on compare avec l'Angleterre (Ballatore, 2010).

En définitive, cet ouvrage fait la part belle à la métaphore et à l'analogie, mais l'image des moyens de transport, employée pour comparer les trois systèmes et les itinéraires étudiants en leur sein, ne saisit qu'imparfaitement les différences évoquées. Peut-on, par exemple, considérer que les wagons de la seconde classe des TGV français sont « surpeuplés » et offrent toujours des « conditions de voyage dégradées », pour caractériser le segment universitaire en France? Il ressort finalement des analyses qu'il n'existe pas d'expérience unique dans chaque pays et que si un principe d'égalité se 
trouve partagé en Europe, c'est bien celui de l'égalité de tous à entrer dans une compétition où personne n'est placé sur un pied d'égalité. Par contre, un des mérites de cet ouvrage est de permettre au lecteur d'observer les décalages existants entre les récits politiques et la réalité sociale dans l'ensemble des trois pays. Il montre également tout l'intérêt qu'il y a à comparer en éducation des réalités territoriales éloignées géographiquement, mais plutôt proches idéologiquement. Largement accessible à un public de non-spécialistes, ce livre a aussi le mérite de mettre à l'honneur les analyses comparatives en éducation, un champ encore fragmenté en Europe où les chercheurs ignorent bien souvent ce qui se fait et s'écrit ailleurs (dans d'autres pays, d'autres langues, voire au sein de différentes institutions issues d'un même pays).

\section{Bibliographie}

Ballatore M. (2010), Erasmus et la mobilité des jeunes Européens, Paris, Presses universitaires de France (" Éducation et société »).

Ballarino G., Bernardi F., Requena M., Schadee H. (2009), «Persistent inequalities? Expansion of education and class inequality in Italy and Spain», European Sociological Review, vol. 25, n 1, p. 123-138.

Ballarino G., Colombo S., Perotti L., Regini M., Semenza R. (2010), « Il mutamento dei rapporti tra università e sistema economico », in R. Moscati, M. Regini, M. Rostan (dir.), Torri d'avorio in frantumi? Dove vanno le università europee, Bologne, il Mulino, p. 31-129.

BERNSTEIN B. (1975), Langage et classes sociales. Codes socio-linguistiques et contrôle social, Paris, Éditions de Minuit (« Le sens commun »).

DUBET F. (2009), Le travail des sociétés, Paris, Seuil.

Duru-Bellat M., Meuret D. (2009), Les sentiments de justice à et sur l'école, Bruxelles, De Boeck Supérieur (« Pédagogies en développement »).

ESPING-ANDERSEN G. (1999), Les trois mondes de l'Etat-providence. Essai sur le capitalisme moderne, Paris, Presses universitaires de France (« Le lien social»).

Felouzis G., Maroy C., Van Zanten A. (2013), Les marchés scolaires, Paris, Presses universitaires de France («Éducation et société »).

GARnEAu S. (2008), «Les mobilités étudiantes en Europe, en Tunisie et au Canada. Processus global et fragmentations sociales», Bulletin d'information de l'Observatoire Jeunes et Société, vol. 7, nº 1, p. 1-8.

Palme M., HultQvist E. (2009), «La transformation de l'école secondaire en Suède : marchandisation, dissolution et reconstruction des frontières institutionnelles et sociales » Recherches en Education, $\mathrm{n}^{\circ} 7$. 
PASQuali P. (2014), Passer les frontières sociales. Comment les «filières d'élite» entrouvrent leurs portes, Paris, Fayard.

TURNeR R. H. (1960), "Sponsored and Contest Mobility and the School System », American Sociological Review, vol. 25, nº 6, pp. 855-867.

VITALE P. (1999), L'enseignement de la sociologie: vers une étude comparative des curricula européens, thèse de doctorat, Marseille, Université d'Aix-Marseille 1.

VITALE P. (2001), «The teaching of Sociology: towards a European comparison of curricula », dans A. Morais, I. Neves, B. Davies, H. Daniels (dir.), Towards a sociology of pedagogy: the contribution of Basil Bernstein to research, New-York, Peter Lang.

VAN ZANTEN A, Kosunen S. (2013), « School choice research in five European countries: the circulation of Stephen Ball's concepts and interpretations », London Review of Education, vol. 11, nº 3, pp. 239-255. 